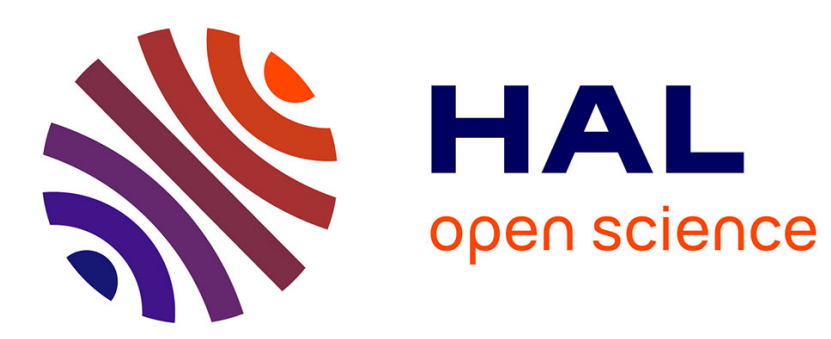

\title{
Health Assessment and Life Prediction of cutting tools based on support vector regression.
}

T. Benkedjouh, Kamal Medjaher, Noureddine Zerhouni, S. Rechak

\section{To cite this version:}

T. Benkedjouh, Kamal Medjaher, Noureddine Zerhouni, S. Rechak. Health Assessment and Life Prediction of cutting tools based on support vector regression.. Journal of Intelligent Manufacturing, 2013, pp.1-19. 10.1007/s10845-013-0774-6 . hal-00867582

\section{HAL Id: hal-00867582 https://hal.science/hal-00867582}

Submitted on 30 Sep 2013

HAL is a multi-disciplinary open access archive for the deposit and dissemination of scientific research documents, whether they are published or not. The documents may come from teaching and research institutions in France or abroad, or from public or private research centers.
L'archive ouverte pluridisciplinaire HAL, est destinée au dépôt et à la diffusion de documents scientifiques de niveau recherche, publiés ou non, émanant des établissements d'enseignement et de recherche français ou étrangers, des laboratoires publics ou privés. 


\title{
Health Assessment and Life Prediction of Cutting tools Based on Support Vector Regression
}

\author{
T. Benkedjouh · K. Medjaher · N. \\ Zerhouni - S. Rechak
}

Received: date / Accepted: date

\begin{abstract}
The integrity of machining tools is important to maintain a high level of surface quality. The wear of the tool can lead to poor surface quality of the workpiece and even to damage of the machine. Furthermore, in some applications such as aeronautics and precision engineering, it is preferable to change the tool earlier rather than to loose the workpiece because of its high price compared to the tool's one. Thus, to maintain a high quality of the manufactured pieces, it is necessary to assess and predict the level of wear of the cutting tool. This can be done by using condition monitoring and prognostics. The aim is then to estimate and predict the amount of wear and calculate the remaining useful life of the cutting tool.

This paper presents a method for tool condition assessment and life prediction. The method is based on nonlinear feature reduction and support vector regression. The number of original features extracted from the monitoring signals is first reduced. These features are then used to learn nonlinear regression models to estimate and predict the level of wear. The method is applied on experimental data taken from a set of cuttings and simulation results are given. These results show that the proposed method is suitable for assessing the wear evolution of the cutting tools and predicting their remaining useful life. This information can then be used by the operators to take appropriate maintenance actions.
\end{abstract}

T. Benkedjouh

EMP, Laboratoire Mécaniques des Structures (LMS), Bordj El Bahri, Algiers, Algeria

K. Medjaher, N. Zerhouni

FEMTO-ST, Université de Franche-Comté/CNRS/ENSMM/UTBM

Automatic Control and Micro-Mechatronic Systems Department

24, rue Alain Savary, 25000 Besançon, France

Tel.: +33 3-81-402796

Fax: +33 3-81-402809

E-mail: kamal.medjaher@ens2m.fr

S. Rechak

ENP, Laboratoire Génie Mécanique, El-Harrach, Algiers, Algeria 
Keywords Tool condition monitoring - feature extraction and reduction · prognostics $\cdot$ remaining useful life $\cdot$ support vector regression

\section{Introduction}

The wear of machining tools can lead to low surface quality and more roughness of the manufactured workpieces. The wear of the tools when it is not detected in time may also lead to damage of the processing machine (or of the tool) and sometimes to accidents. Moreover, the tool wear can impact the reliability, the availability, the security and the quality of the final products. To avoid these situations, it is necessary to monitor the wear level of the tool by developing and implementing appropriate approaches. One of these approaches can be Prognostics and Health Management (PHM). A PHM [13,14,20,29] is a process which involves the following steps: sensors and data acquisition (monitoring), data manipulation (or processing), condition assessment, diagnostics, prognostics and decision support. Concerning the wear of the machining tool, the steps of monitoring, condition assessment and prognostics can be useful to continuously assess and predict the level of wear. Prognostics $[13,14,33,34]$ aims at estimating the remaining useful (RUL) life of a component, sub-system or system [1]. Prognostics can thus be applied on tool condition monitoring to estimate the number of cycles the tool can perform.

The RUL estimation can be done by using three main approaches: model-based prognostics (also called physics of failure prognostics), data-driven prognostics and hybrid prognostics. The model-based approach uses mathematical models including degradation phenomena to estimate the current health status of the system, predict its future one and calculate its RUL. The data-driven approach is based on the utilization of data provided by monitoring sensors to built models in terms of states or trends, which are then used to predict the RUL. Finally, the hybrid approach merges both previous approaches. Modelbased prognostics has the advantage of giving more precise RUL predictions than data-driven prognostics. However, in terms of implementation, the datadriven approach is more easier than the model-based approach. This is because for complex systems, with presence of several components and nonlinearities, it is not trivial to derive their mathematical models. In these cases, data-driven prognostics can be a solution, which makes the trade-off between precision, complexity and cost of implementation.

The cutting process is complex and the relationship between the extracted features from the raw monitoring signals and the observed wear level is nonlinear [21]. Consequently, the application of traditional nonlinear regression methods to estimate and predict the tool wear may be limited. In this domain, Yan and Lee [38] utilized a logistic regression based prognostic method for online performance degradation assessment and failure mode classification of machines. However, the drawback of this method is that it needs various failure pattern data to conduct the logistic regression, which limits its application in real systems. More general than traditional regression models, the support 
vector machine for nonlinear regressions has been successfully applied in various machine learning problems $[27,36]$. However, only few works are published regarding their application in tool condition monitoring. Among these research works, Brezak et al. [6] proposed a continuous hybrid tool wear estimator by using a fuzzy classifier and support vector machines. Dongfeng et al. [28] presented a tool wear predictive model combining least square support vector machines (LS-SVM) and principal component analysis (PCA) technique. Finally, Sun et al. [31] used the SVM in a turning process where the level of wear was classified in a binary class. Thus, the nonlinear feature reduction and nonlinear modeling aspects are not thoroughly addressed in the literature.

This paper deals with the utilization of two nonlinear feature reduction techniques (Expectation-Maximization for Principal Component Analysis: EMPCA [25], and isometric feature mapping: ISOMAP [32]) combined with support vector regression (SVR) models [37] to continuously assess the health state of cutting tools and predict their RUL. The EM-PCA is an enhanced PCA based on iterations and learning for finding the principal subspace. ISOMAP is a manifold learning technique based on pairwise geodesic (or curvilinear) distances derived from high dimensional data. The purpose of EM-PCA and ISOMAP techniques is to build for each set of original features a single health indicator, which can represent the time evolution of the wear of the corresponding cutting tool. The health indicators are then fitted to nonlinear regression models by using the SVR technique [37], which is the most common application form of SVMs.

The proposed method is done in two phases: an offline phase and an online phase. In the first phase, a set of features is extracted from monitoring signals provided by three types of sensors (vibrations, force and acoustic emissions) and related to a set of cutting tools. A Wavelet Packet Decomposition (WPD) [2], particularly the six levels of the db4 taken from the Daubechies family, is utilized to extract features that catch both temporal and frequency information hidden in the raw signals. The features extracted for each cutting tool are then reduced by using the EM-PCA and ISOMAP techniques to one parameter called health indicator. The health indicators are then fitted to nonlinear models by using support vector regressions. The obtained models are finally exploited in the second phase to calculate the RUL of new cutting tools used in the same operating conditions than those learned in the first phase. The proposed method is applied on real data taken from the PHM data challenge 2010 [30].

This paper is organized as follows. Section 2 presents a brief review of condition monitoring and prognostics, section 3 deals with the proposed method for condition assessment of cutting tools and section 4 concerns the application of the method and simulation results. Finally, section 5 concludes the paper. 


\section{Tool condition monitoring and PHM review}

Tool Condition Monitoring (TCM) has been subject of several research works, where different techniques and mathematical tools were used [17,24]. Among these works, Kilundu et al. [16] explored the use of data mining techniques for tool condition monitoring in metal cutting. In their work, the authors highlighted two important aspects: strong relevance of information in high frequency vibration components and benefits of the combination of singular spectrum analysis and band-pass filtering to get rid of useless components (noise). Aliustaoglu et al. [3] developed a tool wear condition monitoring technique based on a two-stage fuzzy logic scheme. In this work, the signals acquired from various sensors were processed to make a decision about the status of the tool. In the same framework, Jemielniak and Arrazola [15] proposed a contribution where acoustic emission and cutting force signals were used for tool condition monitoring in micro-milling of cold-work tool steel. The results obtained revealed a strong influence of tool wear on the acoustic emission signal. $\mathrm{Pu}$ rushothaman [23] proposed a tool condition monitoring in a turning operation by using artificial neural network (ANN). Similarly, Pal et al. [22] developed a neural network based on a sensor fusion model for a tool wear monitoring system in turning operations. Gajate et al. [10] published two model-based approaches for tool wear monitoring on the basis of neuro-fuzzy techniques. In their paper, the use of a neuro-fuzzy hybridization to design a tool wear monitoring system is aimed at exploiting the synergy of neural networks and fuzzy logic, by combining human reasoning with learning and connectionist structure. Always in the category of neural networks, Yeo et al. [40] proposed a novel approach for the estimation of tool wear using the reflectance of cutting chip surface and a back propagation neural network. In their work, the authors postulate that the condition of a tool can be determined by using the surface finish and the color of the cutting chip. Bhattacharyya et al. [5] proposed a method for on-line tool condition monitoring in milling machine by using the current and power signals. In their paper, the authors used a multiple regression model to estimate the tool wear. Alonso et al. [4] developed a reliable tool condition monitoring system for industrial applications based on the vibration analysis of the tool's structure. Chen and Li [8] proposed a technique based on acoustic emission signal wavelet analysis for tool condition monitoring. Finally, Tobon-Mejia et al. [33] presented a contribution for health assessment and remaining useful life of a machining tool in a computer numerical controlled (CNC) machine by using a stochastic approach. More general than TCM, PHM can be considered as a key process for ConditionBased Maintenance (CBM). CBM can be applied to continuously assess the condition of a machining tool and predict its remaining useful life. This latter information can then be exploited to replace the tool before its complete wear. The CBM modules [18] are shown in figure 1. The following of the paper concerns the condition monitoring and assessment and fault prognostic modules. Fault prognostics is defined by the international standard organization as the estimation of the time to failure (ETTF) and the risk of existence or later ap- 


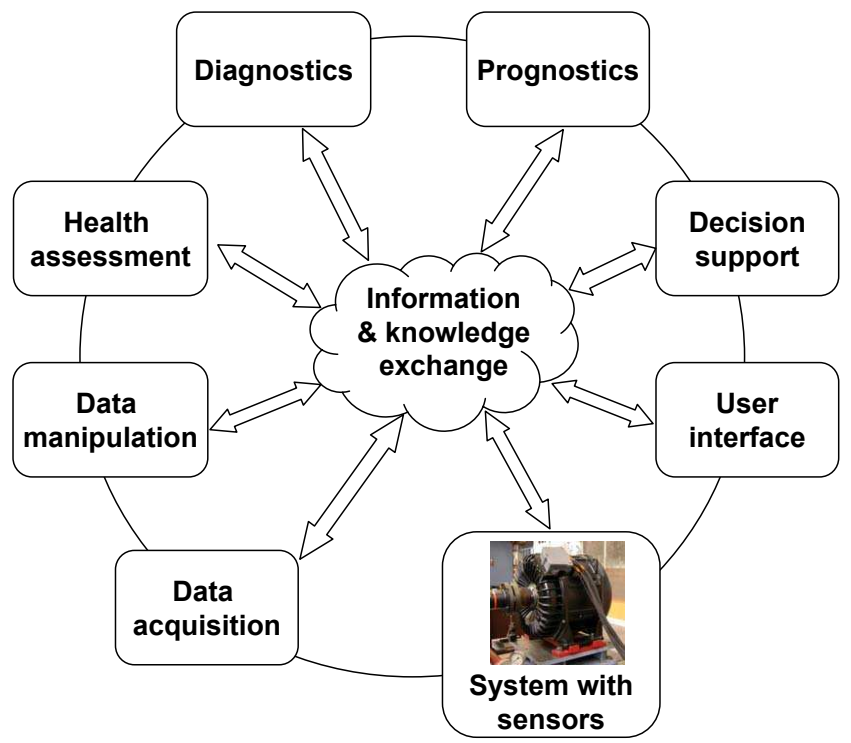

Fig. 1: Modules of a CBM.

pearance of one or more failure modes [1]. However, in the reported research and application works, the terminology ETTF is commonly called Remaining Useful Life (RUL). An illustration of RUL progression is shown in figure 2. Fault prognostics can be done by using three main approaches: model-based,

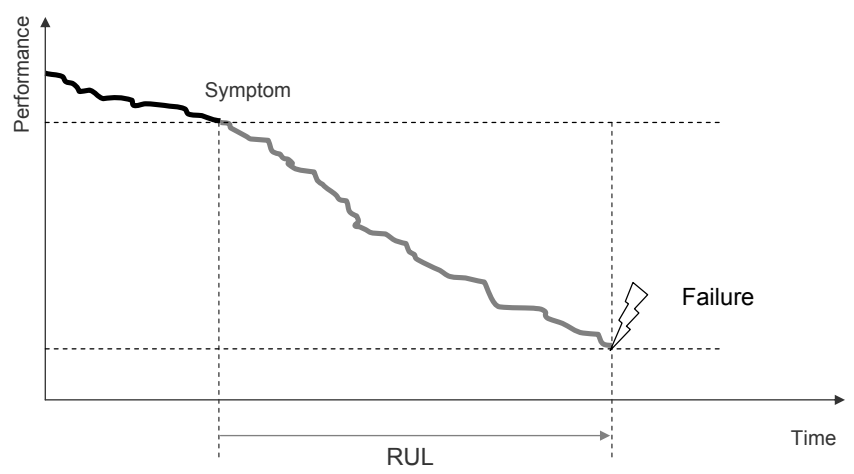

Fig. 2: Illustration of remaining useful life.

data-driven and hybrid prognostics (figure 3).

Model-based (also called physics of failure) methods $[7,11,19]$ deal with the exploitation of a mathematical model representing the behavior of the physical component including its degradation. The derived model is then used to pre- 


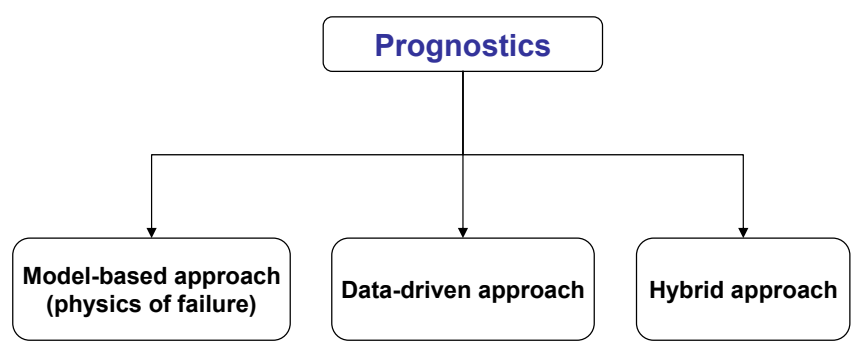

Fig. 3: Fault prognostic approaches.

dict the future evolution of the degradation and to estimate the RUL. In this case, prognostic consists in evolving the degradation model until a determined future instant from the actual deterioration state and by considering the future use conditions of the corresponding component. The main advantage of this approach is its precision, since the predictions are achieved based on a mathematical model including the degradation. However, the derived model is specific to a particular type of component or material, and thus, can not be generalized to all system's components. In addition, getting a mathematical model of degradation phenomena is not an easy task and needs well instrumented test-benches which can be expensive.

Data driven methods $[9,12,20]$ concern the transformation of the monitoring and/or the exploitation data into relevant models, which can be used to assess the health state of the industrial system and predict its future one leading to the estimation of its RUL. The advantage of data-driven prognostic approach is its applicability, cost and implementation. Indeed, by using this approach, it is possible to predict the future evolution of a degradation without any need of prior mathematical model. However, the results obtained by this approach are less precise than those obtained by using model-based methods.

Compared to model-based methods, the data-driven methods offer a trade-off in terms of complexity, cost, precision, and applicability. They are suitable for systems where it is easy to obtain monitoring data and transform them into behavior models of the degradation phenomena. The following of the paper deals with a data-driven prognostic method for cutting tool condition assessment and RUL estimation.

\section{Tool condition assessment and RUL estimation}

The figure 4 shows the steps of the proposed method. A short description of each step is given in the following of the paper.

Cutting tools used for learning: to perform reliable and precise RUL predictions, a set of cutting tools should be used to extract relevant features, build health indicators, fit these indicators to nonlinear regression models, define the fault thresholds, etc. 


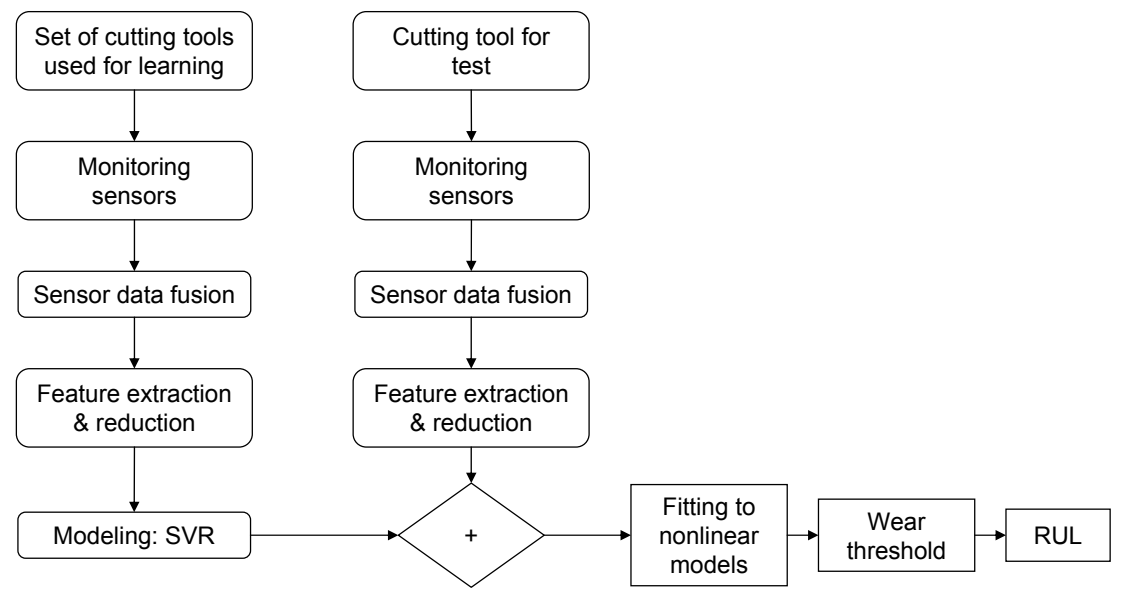

Fig. 4: General scheme of the proposed method.

Monitoring sensors: in TCM and PHM applications, the types and number of sensors to be installed are important and depend on the degradation phenomena to monitor. In this application, one acoustic emission sensor, three accelerometers and three force sensors are used to track the wear evolution of the cutting tools.

Sensor data fusion: the fusion of the data provided by the sensors is important and can help catching more information about the behavior of the wear progression. Indeed, the results obtained by using different sensors can be more precise and more relevant than those issued from the utilization of a single sensor.

Feature extraction and reduction: in practice, different techniques can be used in TCM to extract features from the signals acquired during the utilization of the cutting tools. These features can be temporal (mean, pick to pick, root mean square, etc.), frequency or time-frequency [39]. In this paper, we used the WPD technique [2] (figure 5) to extract the energy coefficients of the six first levels of decomposition. A WPD has special abilities to attain higher discrimination by analyzing the higher frequency domains of a signal. The frequency domains separated by the wavelet packets can be easily selected and classified according to the characteristics of the analyzed signal [2]. A WPD can be seen as a tree where the root is the original signal. The next level of the tree is the result of one step of the wavelet decomposition. Subsequent levels are constructed recursively by applying the wavelet decomposition to the low and high pass filters obtained in the previous level.

The number of original features, corresponding to the energy coefficients of the six first levels of decomposition, is then reduced by using two nonlinear re- 


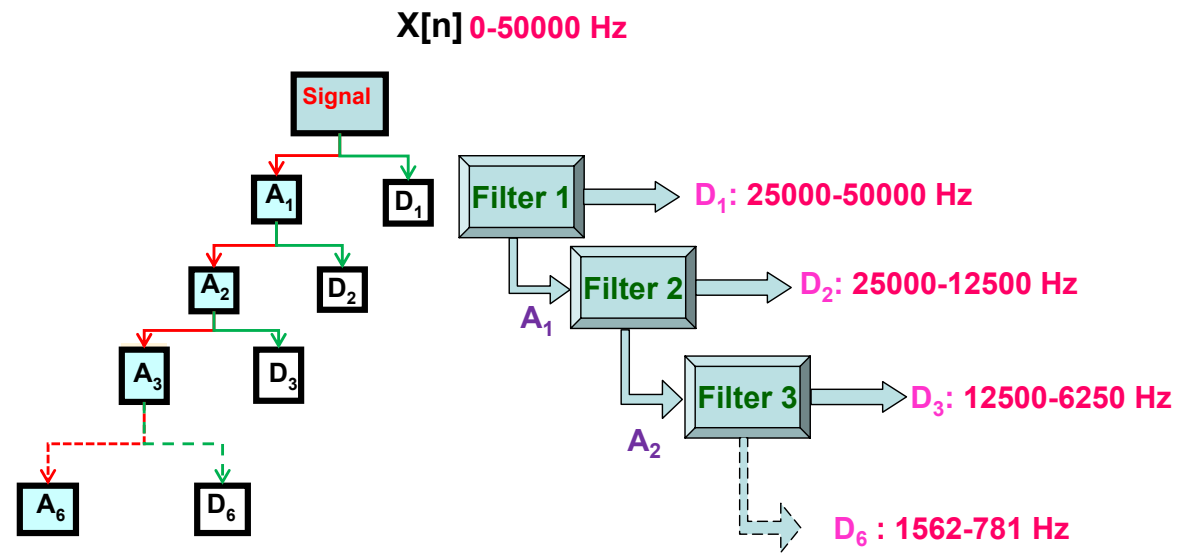

Fig. 5: Wavelet packet decomposition tree.

duction techniques [35]: Expectation-Maximization PCA (EM-PCA) and isometric feature mapping (ISOMAP). The EM-PCA [25] is related to two sets of algorithms: the first is power iteration for solving matrix eigenvalue problems and the second is a competitive learning for finding the principal subspace. ISOMAP [32] is a manifold learning technique based on pairwise distances derived from high dimensional data. The linear techniques such as the principal component analysis and multidimensional scaling (MDS) consider two data points as near points, whereas their distance over the manifold is much larger than the typical inter-points distance. ISOMAP solves this problem by attempting to preserve pairwise geodesic (or curvilinear) distances between data points. The purpose of EM-PCA and ISOMAP techniques is to build for each set of original features a single health indicator, which can represent the time evolution of the wear of the corresponding cutting tool.

SVR modeling: in this step, the health indicators built after reducing the initial features are fitted to nonlinear models by using the SVR technique. The generated models are then used to assess the current health state and calculate the RUL of each cutter. A brief description of SVR technique is presented hereafter.

Support vector machine (SVM) techniques are divided into two categories [36]: support vector classification (SVC) and support vector regression (SVR). SVR is the most common application form of SVMs. It is proposed by Vapnik et al. [37]. The main objective of SVR is to estimate a relationship between input and output random variables under the assumption that the joint distribution of the variables is completely unknown.

A support vector regression (figure 6) can be summarized by the following steps:

- define the data for learning and testing; 
- choose an appropriate kernel function (linear, Gaussian, Radial Basis Function (RBF), polynomial...);

- select an optimal model for training on the input data. The training is done by using a grid research $(\epsilon, C \ldots)$;

- cross-validate on the testing data.
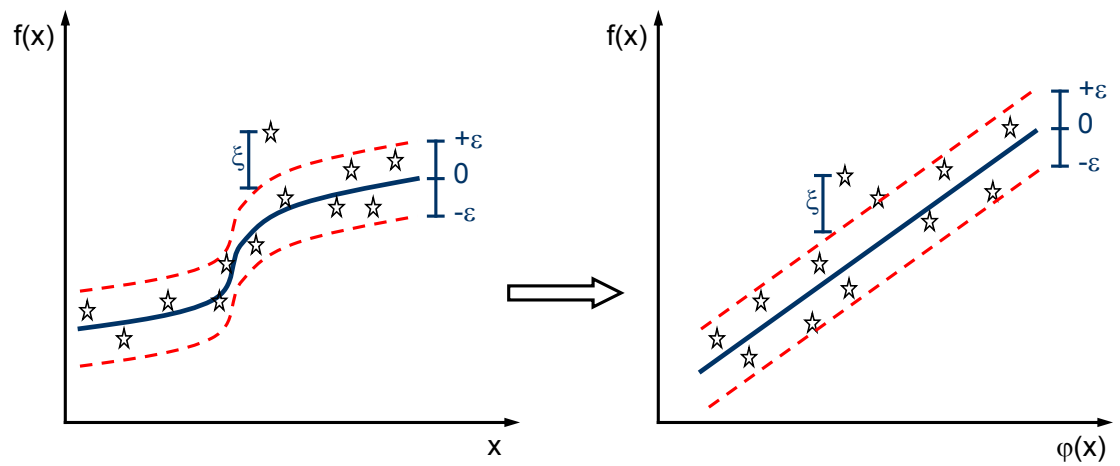

Fig. 6: The principle of SVR for nonlinear regression.

A regression estimation can be formalized as a problem of inferring a function $y=f(x)$ based on the training data $X=\left\{\left(x_{i}, d_{i}\right), i=1, \ldots, l\right\}$, where $x_{i} \in R^{n}$ is the $i^{t h}$ input vector for the $i^{\text {th }}$ training data, $d_{i} \in R$ is the target value for the $i^{t h}$ training data and $l$ is the number of training data. Furthermore, learning a SVR is equivalent to finding a regression function of the form:

$$
f(x)=\sum_{i=1}^{l}\left(\alpha_{i}-\alpha_{i}^{*}\right) k\left(x_{i}, x\right)+b
$$

where $k\left(x_{i}, x\right)$ is a positive definite kernel function, $\alpha=\left(\alpha_{1}, \alpha_{2}, \ldots, \alpha_{l}\right)^{T}, \alpha^{*}=$ $\left(\alpha *_{1}, \alpha *_{2}, \ldots, \alpha *_{l}\right)^{T}$ and $b$ the parameters of the model. The parameters $\alpha_{i}$ and $\alpha_{i}^{*}(i=1, \ldots, l)$ can be calculated by minimizing the following objective function:

$$
\frac{1}{2} \sum_{i, j=1}\left(\alpha_{i}-\alpha_{i}^{*}\right)\left(\alpha_{j}-\alpha_{j}^{*}\right) k\left(x_{i}, x_{j}\right)+\varepsilon \sum_{i=1}^{l}\left(\alpha_{i}+\alpha_{i}^{*}\right)-d \sum_{i=1}^{l}\left(\alpha_{i}-\alpha_{i}^{*}\right)
$$

which is subject to the following constraint:

$$
\sum_{i=1}^{l}\left(\alpha_{i}-\alpha_{i}^{*}\right)=0 \text { and } \alpha_{i}, \alpha_{i}^{*} \in[0, C]
$$

In equations (2) and (3) $\epsilon$ and $C$ are known as the hyper-parameters used to minimize the learning error. The kernel $k\left(x_{i}, x\right)$ of equation $(2)$ can be linear 
or nonlinear. However, the nonlinear kernels give more precise results. In this paper, a Gaussian function which is a nonlinear kernel is used. Its form is given by the following expression:

$$
k\left(x_{i}, x\right)=\exp \left(-\frac{\left\|x_{i}-x\right\|^{2}}{\sigma^{2}}\right)
$$

where $\sigma>0$ is the width of the kernel.

To learn a SVR, one needs to solve the quadratic optimization problem given in equations (2) and (3). With the notion of support vector, the regression function given in equation (1) can be written as follows:

$$
f(x)=\sum_{x_{i} \in S V}^{l}\left(\alpha_{i}-\alpha_{i}^{*}\right) k\left(x_{i}, x\right)+b
$$

In the method proposed in this paper, the regression model given in equation (5) and which is obtained by using the SVR technique is fitted to a nonlinear model. This latter model is then used to calculate the RUL of the cutting tool.

\section{Application and simulation results}

\subsection{Description of the experimental platform}

The experimental platform used to verify the method proposed in the previous section consists of a high speed milling machine (figure 7). This platform

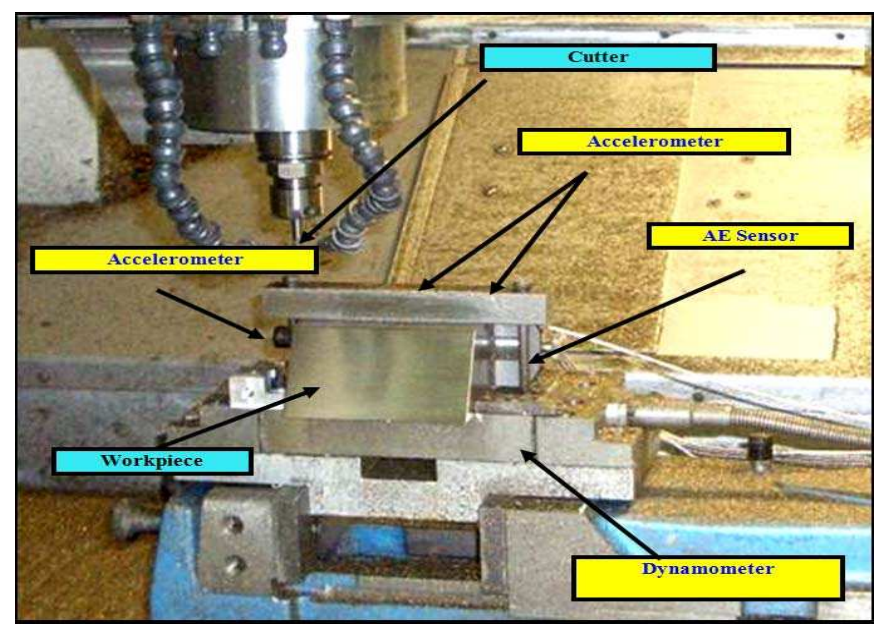

Fig. 7: Experimental setup for tool wear prediction. 


\begin{tabular}{|c|c|c|c|c|}
\hline Spindle speed & Feed rate & $\begin{array}{c}\text { Y cut depth } \\
\text { (radial) }\end{array}$ & $\begin{array}{c}\text { Z cut depth } \\
\text { (axial) }\end{array}$ & $\begin{array}{c}\text { Sampling } \\
\text { frequency }\end{array}$ \\
\hline $10400 \mathrm{RPM}$ & $1555 \mathrm{~mm} / \mathrm{min}$ & $0.125 \mathrm{~mm}$ & $0.2 \mathrm{~mm}$ & $50 \mathrm{kHz}$ \\
\hline
\end{tabular}

Table 1: Cutting conditions.

provides experimental data related to wear of cutting tools used under the operating conditions given in table 1 . The monitoring data are collected from three types of sensors (accelerometers, forces and acoustic emissions) installed on the workpiece. Six individual cutters (C1, C2, C3, C4, C5 and C6), each cutter has three flutes, are used during the experiments conducted on the platform. Each cutter is used for 315 cuts (or cycles) done on the same workpiece and under the same operating conditions. For each cut, the vibrations, force and acoustic emissions are measured and at the end of each cut the wear of the cutters $\mathrm{C} 1, \mathrm{C} 4$ and $\mathrm{C} 6$ is also measured. The acquired data are then stored for later use (315 files corresponding to 315 cuts have been created for each cutter). The experimental platform and the data related to different cuttings are well described in the following reference [30].

\subsection{Simulation results}

The steps of the tool wear condition assessment and prediction method described in section 3 are applied on the experimental data taken from [30]. These data concern three types of sensors: force, vibrations and acoustic emissions. Samples related to first cuts and last cuts are shown in figure 8 .

The first step, after data fusion, of the proposed method concerns feature extraction from the raw signals provided by the three types of sensors (vibration, forcer and acoustic emissions). The features are extracted by using the WPD technique. For this purpose, the detailed energy coefficients of the six first levels of decomposition are calculated from each signal. In this application, the detailed coefficients are preferred to the approximated coefficients because they catch more relevant information about the wear progression of the tools. The energy coefficients extracted from the sensors are shown in figures 9 and 10 .

The extracted features are then reduced by using two nonlinear reduction techniques: EM-PCA and landmark-ISOMAP. The results of this reduction are trends, called health indicators and shown in figure 11. From this latter figure it is possible to observe that the trends have similar progression. However, their magnitudes are different from each other. The obtained health indicators will be correlated with the wear amount progression shown in figure 12 to assess the wear progression and predict the RUL of the cutters.

The trends (like those shown in figure 11) obtained after feature reduction are mapped to nonlinear regressions by using the SVR technique. As stated in section 3, several kernels can be used for SVR. These kernels can be linear, RBF, polynomial, Gaussian, etc. In this paper, after having tested these kernels, we 

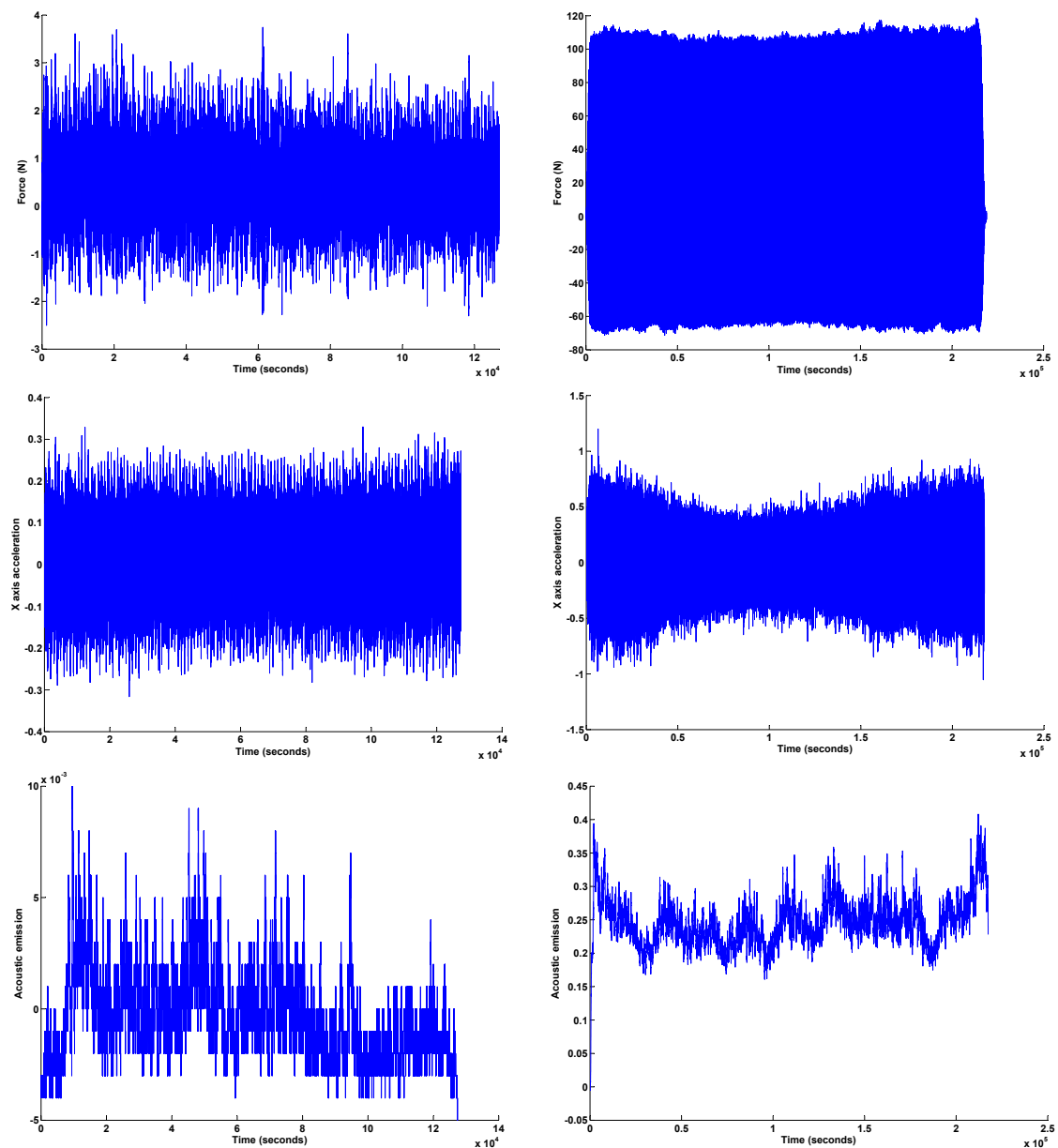

Fig. 8: Samples of force, vibrations and acoustic emissions related to first cut (left) and last cut (right).

found the Gaussian kernel better because it allowed getting better regression models with minimum learning errors. The values of the parameters $C$ and $\epsilon$ depend on the type, the noise and the dimension of the signals or the datasets used for learning. In the case of the application considered in this paper, and after several simulations, the values of the parameters $C$ and $\epsilon$ of the SVR are set equal to 0.00001 and 0.005 , respectively. These values give the optimal learning error for the SVR.

The obtained regressions are then fitted to power models, which are finally used to predict the remaining useful life of each cutter. The power model used 

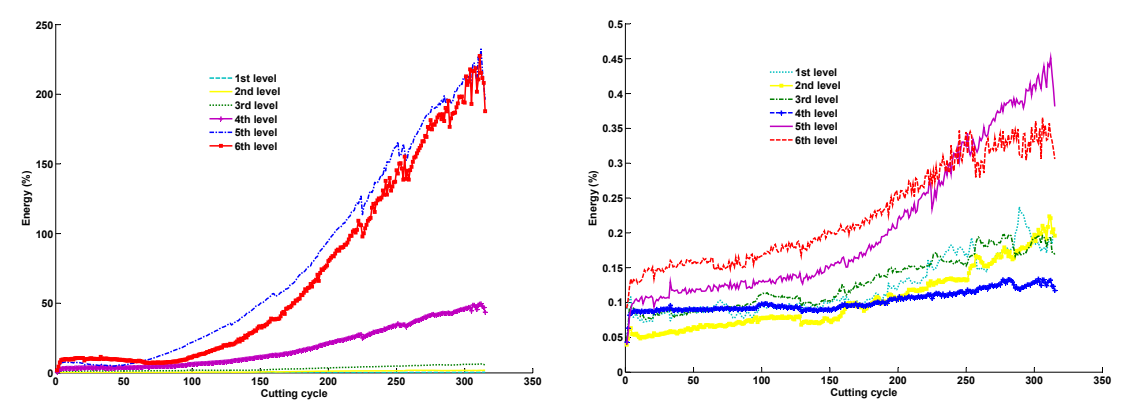

Fig. 9: Energy coefficients extracted from different levels of the force and vibration signals.

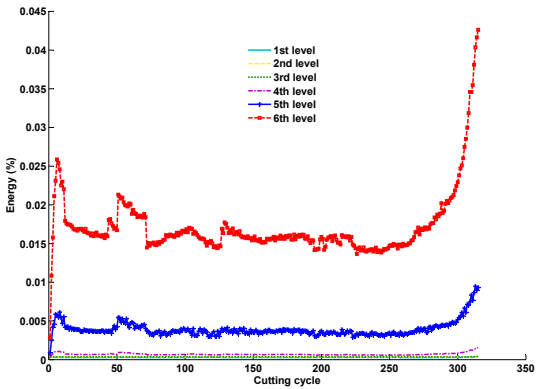

Fig. 10: Energy coefficients extracted from different levels of the acoustic emissions.
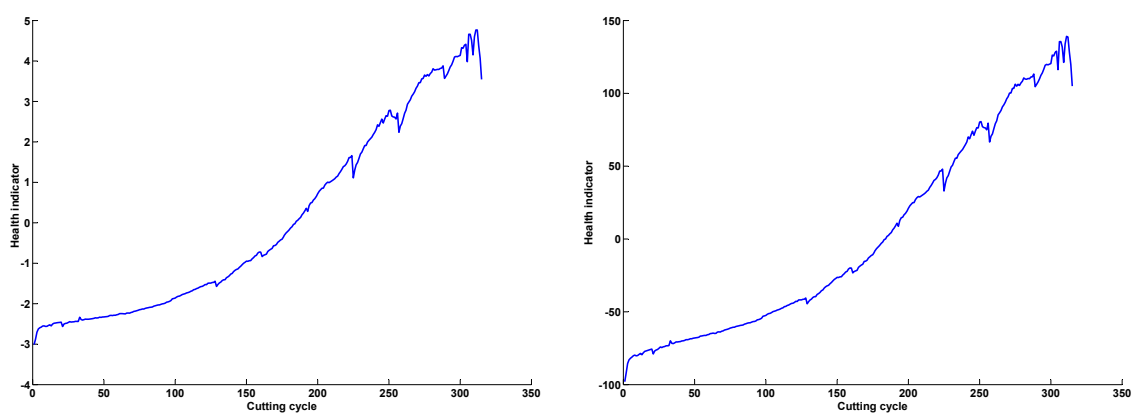

Fig. 11: Result of feature reduction for cutter C1 by using EM-PCA (left) and landmark ISOMAP (right). 

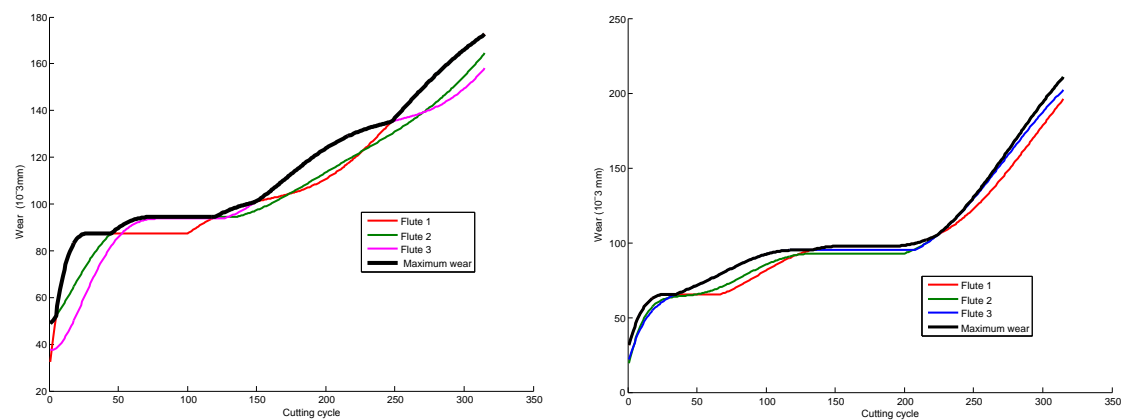

Fig. 12: Wear of three flutes of the cutters $\mathrm{C} 1$ and $\mathrm{C} 4$.
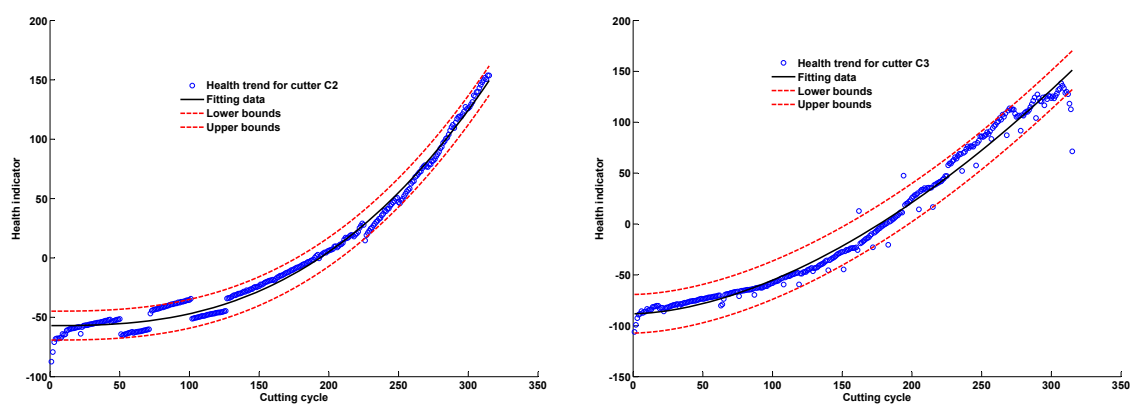

Fig. 13: Regressions and fitting to power models: C2 (left) and C3 (right).

in this application has the following form.

$$
y(t)=a_{0} \cdot t^{a_{1}}+a_{2} \Rightarrow t=\left(\frac{y-a_{2}}{a_{0}}\right)^{\frac{1}{a_{1}}}
$$

The result of regressions obtained for the cutters C2, C3 and C5 are shown in figures 13 and 14. Based on the regressions and the power models given in figure 16, the RUL can then be calculated by using the following expression:

$$
R U L(t)=t_{f}-t
$$

where $t_{f}$ stands for the time for which the tool is out of service (figure 15). The time $t_{f}$ is related to the wear threshold, which is an important parameter during the RUL estimation. In this application, the RUL of each cutting tool is calculated by executing the following steps:

- build the regression model of the corresponding cutting tool;

- define a wear threshold (acceptable limit of wear, as shown in figure 15) and take the corresponding time $t_{f}$;

- calculate the RUL, which is equal to $t-t_{f}$. 


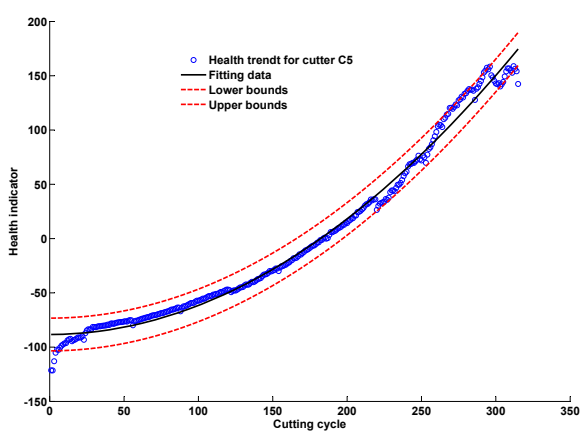

Fig. 14: Regression and fitting to power model for C5.

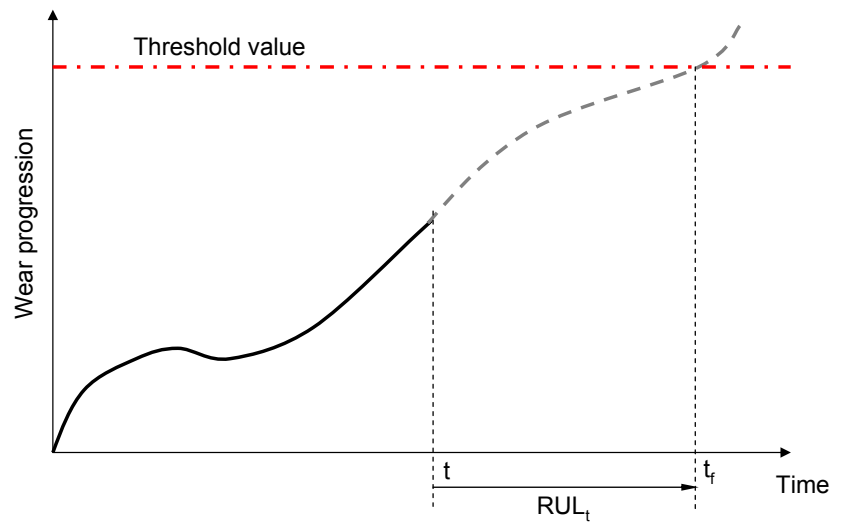

Fig. 15: Calculation of RUL at time t.

\begin{tabular}{|c|c|c|c|}
\hline Cutting tool & Number of cycles & Power regression & R-square \\
\hline C1 & 315 & $y(t)=0.0005397 . t^{1.495}-1.183$ & 0.5107 \\
\hline C2 & 315 & $y(t)=0.1851 . t^{1.325}-162.9$ & 0.5263 \\
\hline C3 & 315 & $y(t)=0.0139 . t^{1.733}-108.6$ & 0.9895 \\
\hline C4 & 315 & $y(t)=0.01925 . t^{1.666}-105.2$ & 0.9768 \\
\hline C5 & 315 & $y(t)=2.665 . t^{0.8514}-193.5$ & 0.4033 \\
\hline C6 & 315 & $y(t)=0.006 . t^{1.85}-93.38$ & 0.9849 \\
\hline
\end{tabular}

Fig. 16: The obtained power models.

In practice, the wear threshold can be given by the expert of the application or obtained experimentally. In this contribution, the threshold corresponds to the amount of wear obtained at the last cutting cycle (315). This value can be changed to correspond to any amount of wear removed from each cutter. However, the way of calculating the RUL will remain the same and can be calculated by using the equation (7). The RUL results corresponding to the 

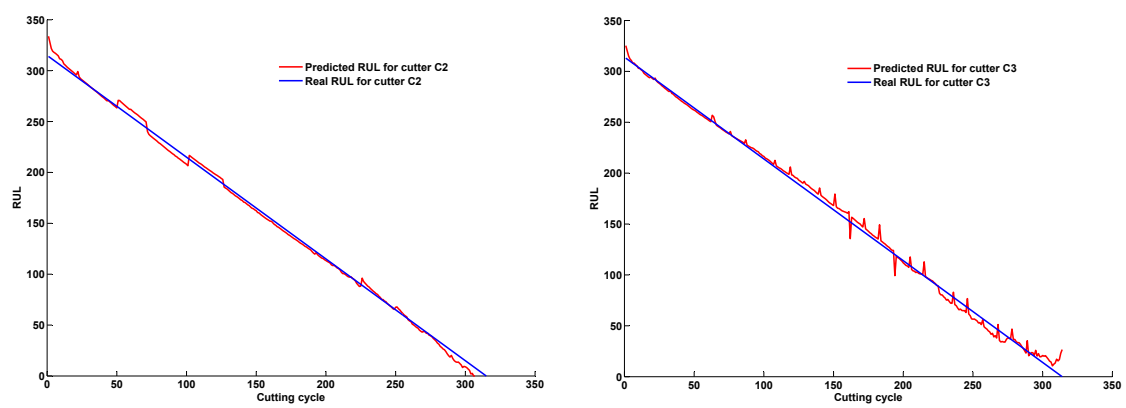

Fig. 17: RUL for the cutters C2 and C3.

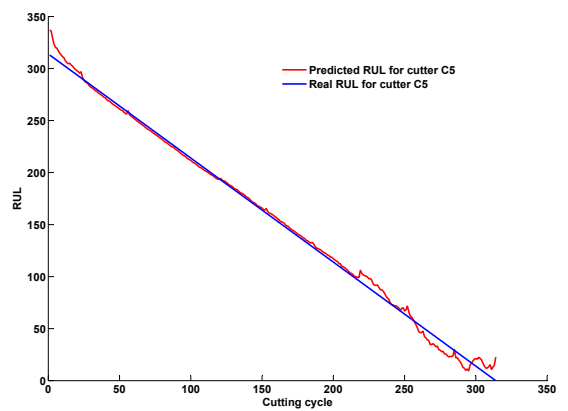

Fig. 18: RUL for the cutter C5.

cutters C2, C3 and C5 are given in figures 17 and 18.

Furthermore, to evaluate the performance of the predictions obtained by the proposed method, the following prognostic metrics are used in this paper: accuracy, precision, Mean Absolute Percentage Error (MAPER) and prognostic horizon [26]. These metrics are briefly introduced hereafter.

- Accuracy: measure of the goodness of the predictions. The accuracy is close to 1 when the predictions are good and far from 1 otherwise.

$$
\text { Accuracy }=\frac{1}{T} \sum_{t=1}^{T} e^{-\frac{\left|R U L_{\text {real }}(t)-R U L(t)\right|}{R U L_{\text {real }}(t)}}
$$

where $R U L_{\text {real }}(t)$ is the real value of RUL, $R U L(t)$ is the predicted RUL at time $t$ and $T$ is the length of the predictions.

- Precision: this measure quantifies the dispersion of the prediction error around its mean.

$$
\text { Precision }=\sqrt{\frac{\sum_{t=1}^{T}(\varepsilon(t)-\bar{\varepsilon})^{2}}{T}}
$$




\begin{tabular}{|c|c|c|c|c|}
\hline Cutting tool & Accuracy & Precision & MAPER & PH \\
\hline C2 & 0.13 & 5.74 & 6.41 & 4 \\
\hline C3 & 0.23 & 10.7 & 8.16 & 9 \\
\hline C5 & 0.51 & 4.59 & 8.98 & 16 \\
\hline
\end{tabular}

Table 2: Results of prognostic metrics.

where $\varepsilon(t)=R U L_{\text {real }}(t)-R U L(t)$ and $\bar{\varepsilon}=\frac{1}{T} \sum_{t=1}^{T} \varepsilon$

- Mean Absolute Percentage Error (MAPER): this measure quantifies the mean error in percentage.

$$
M A P E R=\frac{1}{T} \sum_{t=1}^{T}\left|\frac{100 \cdot \varepsilon(t)}{R U L_{\text {real }}(t)}\right|
$$

- Prognostic Horizon (PH): this measure estimates the time at which the prognostic algorithm gives its first prediction within the confidence interval.

$$
P H=T-t \forall R U L(t) \in\left[R U L_{\text {real }}(t) .\left(1-\alpha_{c}\right), R U L_{\text {real }}(t) .\left(1+\alpha_{c}\right)\right]
$$

with $\alpha_{c} \in[0,1]$

In this application, the implementation of these metrics has led to the results given in table 2. From this table it can be seen that the predictions related to the wear of the cutting tool $\mathrm{C} 2$ are more accurate than those of $\mathrm{C} 3$ and C5. In addition, the prognostic horizon of $\mathrm{C} 2$, i.e. how early the predictions can be done within the confidence level, is better than that of C3 and C5. The predictions of $\mathrm{C} 2$ are good from the cutting cycle number 4 (9 for C3 and 16 for C5).

\section{Conclusion}

This paper presented a method for tool wear assessment and RUL prediction by using two nonlinear feature reduction techniques and support vector regression. The method is applied on experimental data provided by a set of cuttings done on milling machine. Several sensors were installed on the workpiece to catch the wear progression. A set of features were extracted from these signals by using the wavelet packet decomposition technique. The features consisted of the detailed energy coefficients extracted from the first six levels of decomposition. The set of initial features related to each cutting tool is then reduced by using the EM-PCA and landmark-ISOMAP techniques to one combined feature considered as a health indicator. This latter indicator is mapped to a nonlinear regression by using SVR and the obtained regression is fitted to a power model. This model is finally used to estimate the wear progression and predict the remaining useful life of the cutting tools. Indeed, the wear estimation and prediction are relevant information that the operator can use to perform reliable machining works. 


\section{References}

1. AFNOR: Condition monitoring and diagnostics of machines - prognostics - part 1: General guidelines. NF ISO 13381-1 (2005)

2. Akansu, A.N., Serdijn, W.A., Selesnick, I.W.: Emerging applications of wavelets: A review. Physical Communication 3(1), 1 - 18 (2010)

3. Aliustaoglu, C., Ertunc, H.M., Ocak, H.: Tool wear condition monitoring using a sensor fusion model based on fuzzy inference system. Mechanical Systems and Signal Processing 23, 539 - 546 (2009)

4. Alonso, F., Salgado, R.: Analysis of the structure of vibration signals for tool wear detection. Mechanical Systems and Signal Processing 22, 735 - 748 (2008)

5. Bhattacharyya, P., Sengupta, D., Mukhopadhyay, S., Chattopadhyay, A.B.: Online tool condition monitoring in face milling using current and power signals. International Journal of Production Research 46(4), 1187 - 1201 (2008)

6. Brezak, D., Majetic, D., Udiljak, T., Kasac, J.: Tool wear estimation using an analytic fuzzy classifier and support vector machines. Journal of Intelligent Manufacturing $\mathbf{2 3}$ $797-809(2012)$

7. Chelidze, D., Cusumano, J.: A dynamical systems approach to failure prognosis. Journal of Vibration and Acoustics 126, 2 - 8 (2004)

8. Chen, X., Li, B.: Acoustic emission method for tool condition monitoring based on wavelet analysis. International Journal of Advanced Manufacturing Technology 33, 968 - 976 (2007)

9. Dong, M., He, D.: A segmental hidden semi-markov model (hsmm)-based diagnostics and prognostics framework and methodology. Mechanical Systems and Signal Processing 21, 2248-2266 (2007)

10. Gajate, A., Haber, R., Del Toro, R., Vega, P., Bustillo, A.: Tool wear monitoring using neuro-fuzzy techniques: a comparative study in a turning process. Journal of Intelligent Manufacturing 23, 869-882 (2012)

11. He, D., Li, R., Bechhoefer, E.: Stochastic modeling of damage physics for mechanical component prognostics using condition indicators. Journal of Intelligent Manufacturing 23, $221-226(2012)$

12. Heng, A., Tan, A.C., Mathew, J., Montgomery, N., Banjevic, D., Jardine, A.K.: Intelligent condition-based prediction of machinery reliability. Mechanical Systems and Signal Processing 23(5), 1600 - 1614 (2009)

13. Heng, A., Zhang, S., Tan, A.C.C., Mathew, J.: Rotating machinery prognostics: state of the art, challenges and opportunities. Mechanical Systems and Signal Processing 23(3), $724-739$ (2009)

14. Jardine, A.K., Lin, D., Banjevic, D.: A review on machinery diagnostics and prognostics implementing condition-based maintenance. Mechanical Systems and Signal Processing $\mathbf{2 0}(7), 1483-1510(2006)$

15. Jemielniak, K., Arrazola, P.: Application of ae and cutting force signals in tool condition monitoring in micro-milling. CIRP Journal of Manufacturing Science and Technology 1, $97-102(2008)$

16. Kilundu, B., Dehombreux, P., Chiementin, X.: Tool wear monitoring by machine learning techniques and singular spectrum analysis. Mechanical Systems and Signal Processing 25, $400-415(2011)$

17. Kunpeng, Z., San, W.Y., Soon, H.G.: Wavelet analysis of sensor signals for tool condition monitoring: A review and some new results. International Journal of Machine Tools and Manufacture 49, 537 - 553 (2009)

18. Lebold, M., Thurston, M.: Open standards for condition-based maintenance and prognostic systems. In: Proc. 5th Maintenance and Reliability Conference (MARCON) (2001)

19. Luo, J., Pattipati, K.R., Qiao, L., Chigusa, S.: Model-based prognostic techniques applied to a suspension system. Transactions on Systems, Man, and Cybernetics 38, 1156-1168 (2003)

20. Medjaher, K., Tobon-Mejia, D.A., Zerhouni, N.: Remaining useful life estimation of critical components with application to bearings. IEEE Transactions on Reliability 61(2), $292-302$ (2012) 
21. Oraby, S., Hayhurst, D.: Cutting tool condition monitoring using surface texture via neural network. Int. J. of M/c Tools Mfg. 44, $1261-1269$ (2004)

22. Pal, S., Heyns, P.S., Freyer, B.H., Theron, N.J., Pal, S.K.: Tool wear monitoring and selection of optimum cutting conditions with progressive tool wear effect and input uncertainties. Journal of Intelligent Manufacturing 22, 491-504 (2011)

23. Purushothaman, S.: Tool wear monitoring using artificial neural network based on extended kalman filter weight updation with transformed input patterns. Journal of Intelligent Manufacturing 21, 717-730 (2010)

24. Rehorn, A., Jiang, J., Orban, P.: State of the art methods and results in tool condition monitoring: a review. International Journal of Advance Manufacturing Technology 26 693-710 (2005)

25. Roweis, T., Saul, L.: Nonlinear dimensionality reduction by locally linear embedding. Science 290, $2323-2326$ (2000)

26. Saxena, A., Celaya, J., Saha, B., Saha, S., Goebel, K.: Metrics for offline evaluation of prognostic performance. International Journal of Prognostics and Health Management (2010)

27. Schölkopf, B., Smola, A.: Learning with kernels. MIT Press (2002)

28. Shi, D., Gindy, N.N.: Tool wear predictive model based on least squares support vector machines. Mechanical Systems and Signal Processing 21(4), 1799 - 1814 (2007)

29. Sikorska, J., Hodkiewicz, M., Ma, L.: Prognostic modelling options for remaining useful life estimation by industry. Mechanical Systems and Signal Processing 25(5), 1803 1836 (2011)

30. PHM Society: PHM data chalelnge 2010. https://www.phmsociety.org/competition/phm/10 (2010)

31. Sun, J., Hong, G., Rahman, M., Wong, Y.: The application of nonstandard support vector machine in tool condition monitoring system. In: Second IEEE International Workshop on Electronic Design, Test and Applications DELTA 2004, pp. 295 - 300. (2004)

32. Tenenbaum, J., Silva, V.D., Langford, J.C.: A global geometric framework for nonlinear dimensionality reduction. Science 290, 2319 - 2323 (2000)

33. Tobon-Mejia, D.A., Medjaher, K., Zerhouni, N.: Cnc machine tool's wear diagnostic and prognostic by using dynamic bayesian networks. Mechanical Systems and Signal Processing 28, 167 - 182 (2012)

34. Vachtsevanos, G., Lewis, F.L., Roemer, M., Hess, A., Wu, B.: Intelligent fault diagnosis and prognosis for engineering systems. Wiley (2006)

35. Van Der Maaten, L.J.P., Postma, E.O., Van Den Herik, H.J.: Dimensionality reduction: A comparative review. Tech. rep., Tilburg University (2009). TiCC-TR 2009-005

36. Vapnik, V.: The Nature of Statistical Learning Theory. Springer, New York (1995)

37. Vapnik, V., Golowich, S., Smola, A.: Support vector method for function approximation, regression estimation, and signal processing. Advances in Neural Information Processing Systems 9, $281-287$ (1997)

38. Yan, J.H., Lee, J.: Degradation assessment and fault modes classification using logistic regression. Journal of Manufacturing Science and Engineering Transactions of the ASME 127(4), 912-914 (2005)

39. Yan, W., Qiu, H., Iyer, N.: Feature extraction for bearing prognostics and health management (PHM)-a survey. Tech. rep., Air Force Research Laboratory (2008)

40. YEO, S.H., KHOO, L.P., NEO, S.S.: Tool condition monitoring using reflectance of chip surface and neural network. Journal of Intelligent Manufacturing 11, 507-514 (2000) 